\title{
Comparación de algunos métodos de procesamiento para Fonsecaea pedrosoi al microscopio electrónico de rastreo
}

\author{
José B. Yung ${ }^{1}$, María C. Cepero², Francisco J. Leal ${ }^{3}$
}

\begin{abstract}
Resumen
Se evaluaron cuatro métodos de procesamiento para el estudio ultraestructural de hongos al microscopio electrónico de rastreo (MER). Se utilizaron microcultivos de cepas colombianas de Fonsecaea pedrosoi aisladas de lesiones de pacientes en el Instituto Dermatológico Federico Lleras Acosta, Santafé de Bogotá. Los resultados mostraron alteraciones, distorsiones 0 artificios en los hongos procesados por tres de los métodos utilizados, debido probablemente a los efectos físicos o químicos durante el procesamiento. Se propone el método de fijación con vapores de acroleína pura y cristales de $\mathrm{OsO}_{4}$ con deshidratación y secado en alto vacío que evitó la pérdida de estructuras y conservó los arreglos característicos, presentando rapidez, fácil manipulación del especimen y disminución de reactivos. Se destaca la importancia del MER en el estudio morfológico, estructural y taxonómico de los hongos y el uso de los vapores de acroleína para la fijación y el alto vacío para el secado, como alternativas metodológicas para el procesamiento de especímenes biológicos de difícil manipulación o que presentan fácil alteración por su composición higrófila.
\end{abstract}

\section{Summary}

Some conventional procedural methods were evaluated for the ultrastructural study of fungi by scanning electron microscope (SEM). Microcultures of Colombian strains of Fonsecaea pedrosoi isolated from patients injuries from the Federico Lleras Acosta Dermatological Institute, Santafé de Bogotá, Colombia, were used. The results showed alterations, distortions or artifacts on the processed fungi by three of the methods used, probably due to physical or chemical effects during the processing. Fixation with pure acrolein vapors and $\mathrm{OsO}_{4}$ crystals with dehydration and high vacuum drying, avoided the loss of some structures and characteristic arrangements, was also fast, provided easy manipulation of the specimen and needed less reagents. The importance of the SEM is emphasized by the morphological, structural and taxonomical study of the fungi: the use of acrolein vapors for fixation and high vacuum drying as methodological alternatives for the processing of biological samples which are difficult to manipulate or that present easy alteration due to their high hydrophillic composition.

\footnotetext{
1 Laboratorio de Investigación Clínica, Departamento de Ciencias Biológicas, Universidad de Los Andes; Laboratorio de Inmunología, Hospital de San José, Santafé de Bogotá, D.C.

2 Departamento de Ciencias Biológicas, Centro de Investigaciones Microbiológicas, Universidad de Los Andes, Santafé de Bogotá, D.C.

3 Unidad de Alergia e Inmunología, Hospital Infantil Universitario Lorencita Villegas de Santos, Santafé de Bogotá, D.C.
} 
La cromomicosis es una micosis crónica y cosmopolita que afecta la piel y los tejidos subcutáneos (1), que ocasiona grandes lesiones polimórficas de aspecto verrucoso, costroso, nodulares, ulceradas o cicatriciales (2). Losagentes etiológicos en la mayoría de las infecciones son: Fonsecaea pedrosoi, Fonsecaea compactum, Phialophora verrucosa, Cladosporium carrioniiy Rhinocladiella aquaspersa (3). Estos hongos tienen el mismo aspecto macroscópico en cultivo y sólo se diferencian por su modo de conidiación (4).

Para la identificación correcta de los hongos dematiáceos, las características microscópicas siguen siendo las más importantes, por lo cual se necesita estudiar y analizar las estructuras de esporulación sin las alteraciones que ocurren en las preparaciones para la observación al microscopio de luz.

Se han realizado grandes e importantes estudios que incluyen observaciones de diferentes cepas de $F$. pedrosoi al microscopio de luz $(5,6)$ y al microscopio electrónico de rastreo (MER) (7); sin embargo, en ninguno de estos trabajos se estudian cepas colombianas y al MER sólo se ha descrito la conidiación tipo Cladosporium.

Este trabajo pretende evaluar cuatro métodos de procesamiento para hongos al MER y describir ultraestructuralmente las características morfológicas de conidiación de dos cepas colombianas de $F$. pedrosoi a partir de microcultivos.

\section{Materiales y métodos}

Hongos: se utilizaron dos cepas colombianas de F. pedrosoi (C-880957, C-2569) aisladas de lesiones de pacientes que consultaron al Instituto Dermatológico Federico LLeras Acosta de Santafé de Bogotá e identificadas en el Centro de Investigaciones Microbiológicas de la Universidad de Los Andes. Los hongos se mantuvieron por repiques sucesivos en medio Sabouraud hasta su procesamiento.

Cultivos: los hongos se subcultivaron en Sabouraud a $22^{\circ} \mathrm{C}$. De cada cepa se realizaron 15 microcultivos en papa dextrosa agar (PDA) utilizando una placa metálica pequeña estéril que sirvió de soporte y base para el procesamiento al $\operatorname{MER}(8,9)$.
Procesamiento al MER: las placas metálicas con el hongo sobre la cara inferior se desprendieron del microcultivo. Se procesaron varias láminas de cada cepa por cada uno de los siguientes cuatro métodos a evaluar (comunicación personal de Francisco Hernández Chavarría, Universidad de Costa Rica, Unidad de Microscopía Electrónica).

En el método 1, los hongos que crecieron sobre la lámina metálica se fijaron por $2 \mathrm{~h}$ con vapores de $\mathrm{OsO}_{4}$ al $4 \%$ en amortiguador fosfato (AF) $0,1 \mathrm{M}, \mathrm{pH} 7,2-7,4$; luego, se pasaron por etanol al $70 \%$ para disminuir la hidrofobicidad y, posteriormente, se realizó una postfijación con glutaraldehído al $2,5 \%$ en AF durante $2 \mathrm{~h}$ a $4^{\circ} \mathrm{C}$. Después, se lavaron tres veces con AF y dos veces con agua destilada; se deshidrataron a temperatura ambiente en concentraciones ascendentes de etanol durante $20 \mathrm{~m}$ en cada uno y se secaron en punto crítico y terbutanol.

En el método 2, los especímenes se fijaron con vapores de $\mathrm{OsO}_{4}$ al $1 \%$ en $\mathrm{AF}$ por $24 \mathrm{~h}$ a temperatura ambiente; se sometieron a un enfriamiento rápido con nitrógeno líquido y se postfijaron con vapores de $\mathrm{OsO}_{4}$ al $6 \%$ en acetona a bajas temperaturas: a $-70^{\circ} \mathrm{C}$ y $-20^{\circ} \mathrm{C}$ por $72 \mathrm{~h}$, a $-4^{\circ} \mathrm{C}$ por $12 \mathrm{~h}$. Después de la postfijación se deshidrataron en acetona durante 30 minutos y se secaron en terbutanol.

En el método 3, las cepas se fijaron con solución de Karnovsky pH 7,2 por $12 \mathrm{~h}$ a temperatura ambiente; se lavaron tres veces con AF; se sumergieron en ácido tánico acuoso al $1 \%$ por $2 \mathrm{~h}$; se lavaron tres veces con AF por $10 \mathrm{~m}$; se postfijaron con vapores de $\mathrm{OsO}_{4}$ al $1 \%$ por $2 \mathrm{~h}$; se lavaron tres veces por $3 \mathrm{~m}$ con agua destilada; se deshidrataron a concentraciones ascendentes de etanol hasta etanol absoluto durante $20 \mathrm{~m}$ a temperatura ambiente y se secaron en punto crítico.

En el método 4, los hongos se sometieron a vapores de acroleína pura por $3 \mathrm{~h}$; se postfijaron con cristales de $\mathrm{OsO}_{4}$ por $4 \mathrm{~h}$; se secaron y cubrieron con $200 \AA$ de oro en un cobertor iónico (EIKO IB-3) por $2 \mathrm{~m}$ (0,05 Torr, $10 \mathrm{~mA}, 350 \mathrm{Kv})$ (comunicación personal de Yutaka Futaesaku, Ph. D., Universidad de Kitasato, Japón). 
Secado: para el secado de punto crítico, en los métodos 1 y 3 , se utilizó la mezcla etanol- $\mathrm{CO}_{2}$ evacuando a una velocidad de $1 \mathrm{~L} / \mathrm{m}$ (Hitachi HCP-2) (10). El secado por terbutanol en los métodos 1 y 2 se realizó por congelaciónsublimación con terbutanol (Freeze drying VFD20) (11).

Montaje y observación al MER: los hongos secados se observaron en un microscopio estereoscópico y se colocaron pequeños puentes de pintura de plata entre el hongo y la superficie de la lámina metálica. Se cubrieron con una ultracapa de oro de $200 \AA$ en un cobertor iónico (EIKO IB3) $(0,05$ Torr, $10 \mathrm{~mA}, 350 \mathrm{Kv})$ por $2 \mathrm{~m}$. Las observaciones se hicieron en el MER (Hitachi S570 ) a $15 \mathrm{Kv}$ con una resolución de $30 \AA \AA$. Las microfotografías se tomaron a una distancia de trabajo entre 1 y $20 \mathrm{~mm}$, a una magnificación entre $1.000 \times$ y $15.000 \mathrm{X}$, utilizando película Kodak VP-120 (Verichrome pan film) ISO 125.

\section{Resultados}

Los tres primeros métodos revelaron alteraciones en las estructuras fúngicas que impidieron estudiar la ultraestrutura de los hongos. El método 1 mostró colapso de las hifas (micrografías 1 y 2) y de la célula conidiógena tipo fiálide (micrografía 3); artefactos sobre las hifas, fiálides y conidios (micrografías 1 y 3 ) que probablemente correspondan a artificios derivados del procesamiento o a restos del medio de cultivo. Los métodos 2 (micrografías 4 y 5 ) y 3 (micrografías 6 y 7) presentaron alteraciones y distorsiones morfológicas en los hongos, similares a las observadas en el primer método. Sin embargo, en algunas observaciones se aprecia ultraestructuralmente el colarete en las fiálides (micrografías 3, 6 y 7). En contraste, el método 4 permitió estudiar con detalle los diferentes tipos de conidiación: fiálide (micrografía 8), Cladosporium (micrografías 9a 12) y Rhinocladiella (micrografías 13 y 14).

En la micrografía 8 se aprecia con detalle la esporulación tipo fiálide. Nótese el arreglo de los conidios en falsa cabeza y la unión de estos fialoconidios por puentes de aspecto mucilaginoso.

Las micrografías 9 y 10 muestran la esporulación tipo Cladosporium donde los conidios primarios se producen en forma holoblástica de dentículos en un ápice irregular e hinchado de una célula conidiógena; obsérvese en la micrografía 9, un conidio secundario naciendo de un conidio primario, sucesión acropétala.

En este tipo de conidiación se observa también un arreglo asimétrico en donde células de diferente tamaño con diversos estados de desarrollo nacen a partir del mismo conidióforo, característico de la conidiogénesis blástica (micrografías 9 a 11), apreciándose en algunos el comienzo del arreglo catenulado en el cual el conidio más joven se encuentra al extremo de la cadena (micrografía 12).
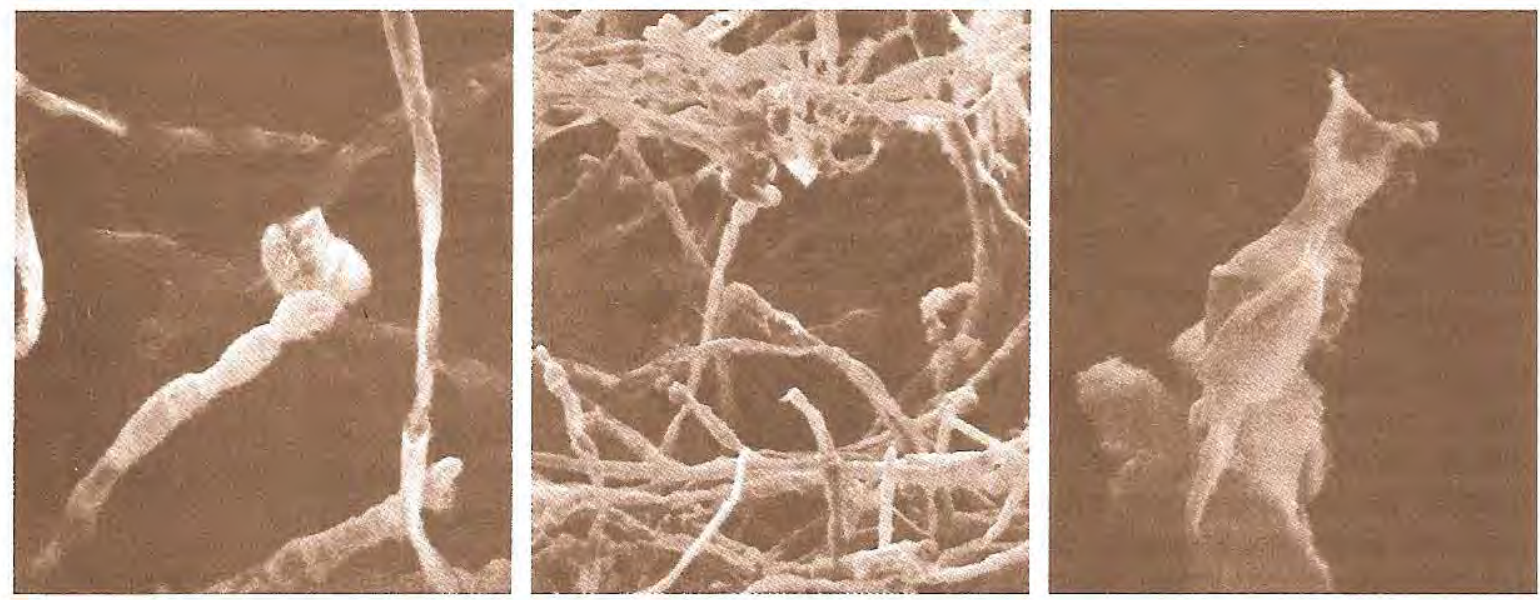

Micrografías 1-3. Cepas de Fonsecaea pedrosoi procesadas por el método $1 ; 1$ y 3 secadas en punto crítico y la 2 con terbutanol. 

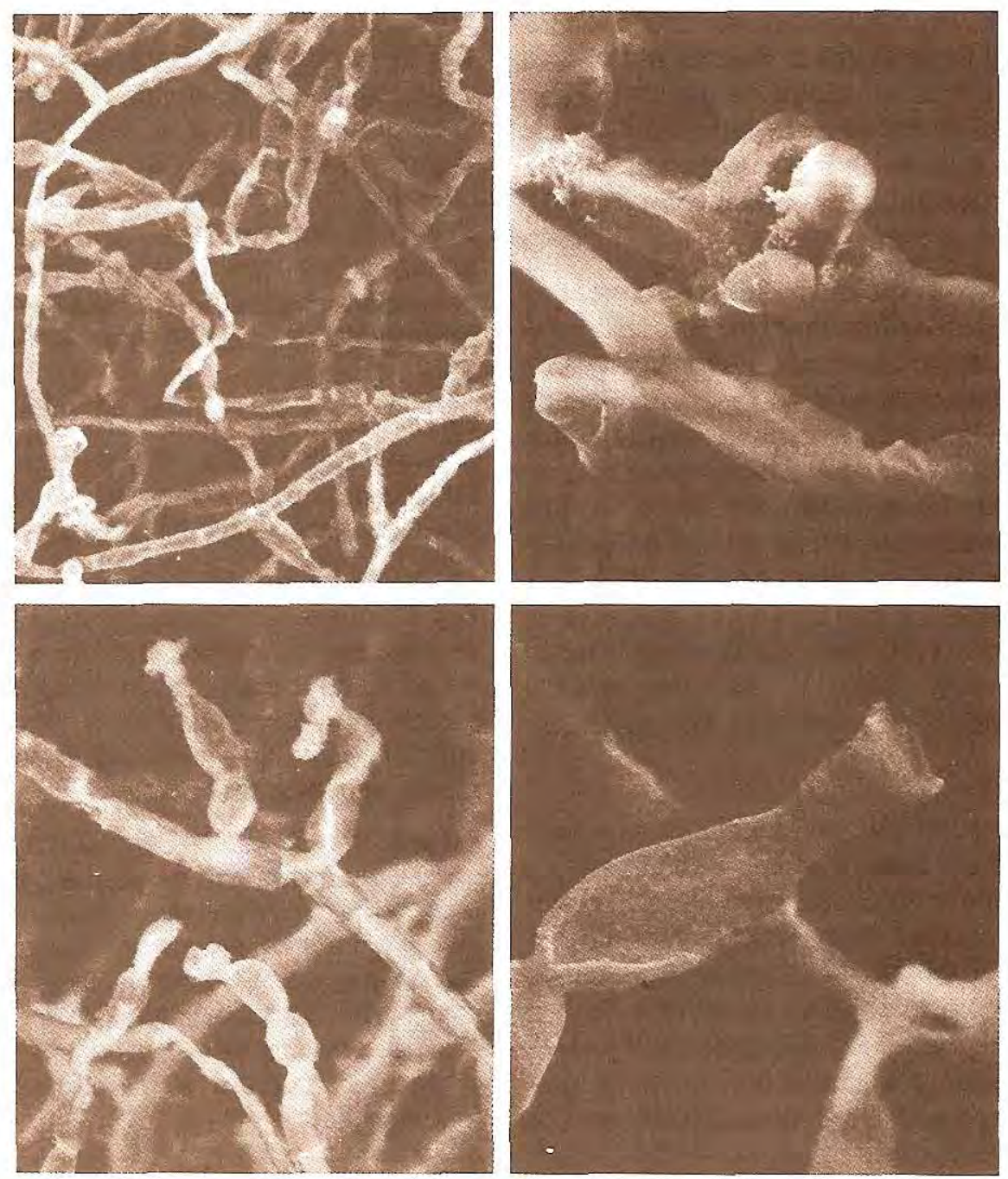

Micrografías 4-7. Cepas de Fonsecaea pedrosoi, 4 y 5 procesadas por el método $2 ; 6$ y 7 por el método 3 ; secadas en punto crítico y terbutanol.

En la micrografía 13, arreglo derecho, se muestra una conidiación similar al tipo Rhinocladiella con conidios esféricos de diferente tamaño nacidos de una célula conidiógena ligeramente ampuliforme.

La cepa C-2569 mostró una célula conidiógena que al microscopio de luz se vería como una fiálide (micrografía 14), pero que con el MER mostró ser de mayor tamaño, piriforme, con extremo basal redondeado, de superficie lisa, ápice hinchado, con varios dentículos en el extremo apical, ausencia de colarete y en la prolongación simpodial dos conidios primarios ovoides.
Nótese al fondo, en esta misma micrografía, una célula de morfología similar, pero que claramente es de tipo fiálide con colarete notorio.

La cepa C-2569 mostró un arreglo de superficie lisa y piriforme (micrografía 15) y la C-880957 mostró un arreglo poco característico a manera de célula conidiógena lisa, piriforme, con un cuello o prolongación, observándose en ella varios dentículos (micrografía 16).

Estos últimos arreglos no se correlacionan con ninguna de las observaciones descritas al microscopio de luz o al microscopio de contraste de fase. 

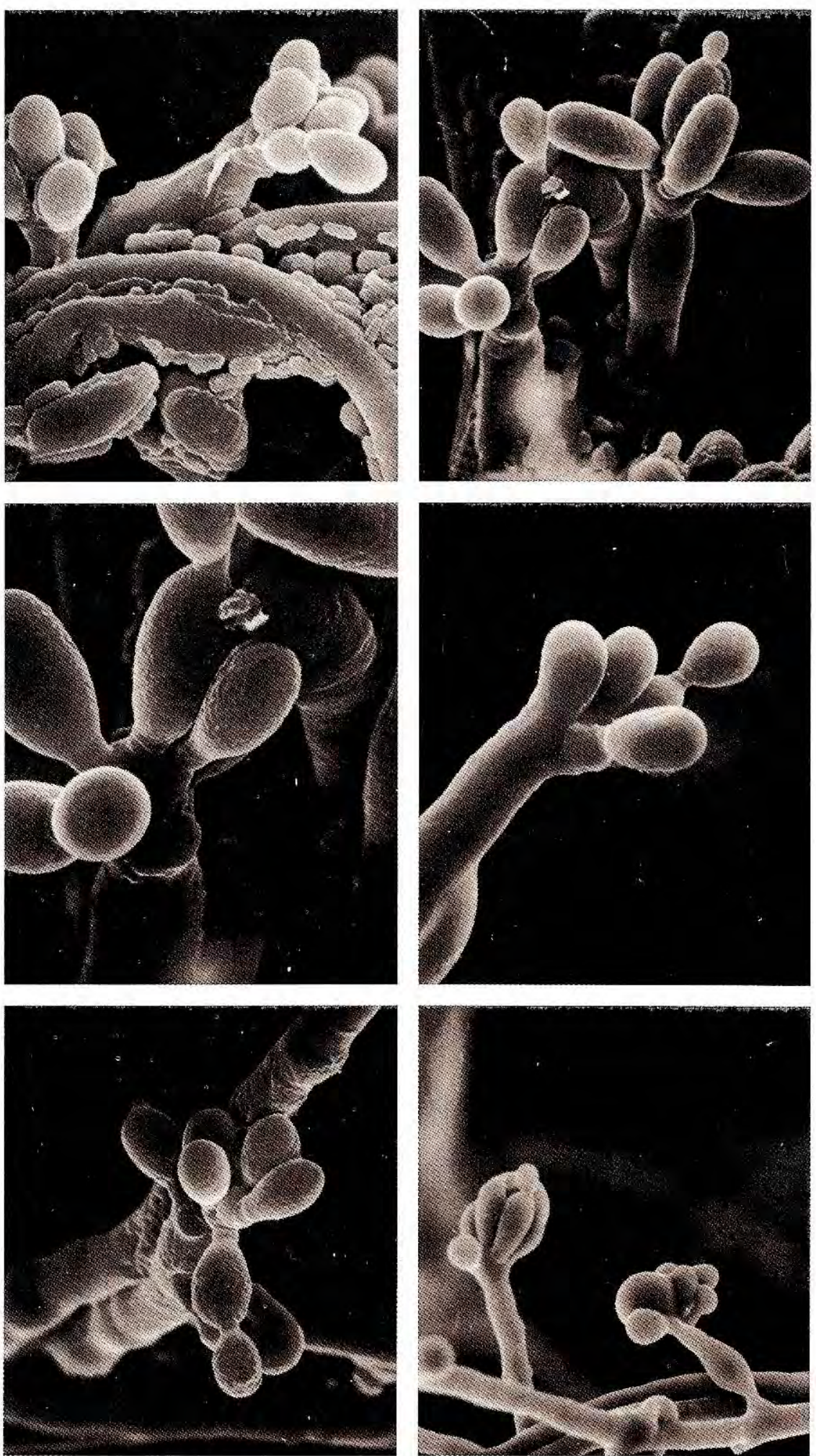

Micrografías 8-13. Cepas de Fonsecaea pedrosoi procesadas por el método 4. 

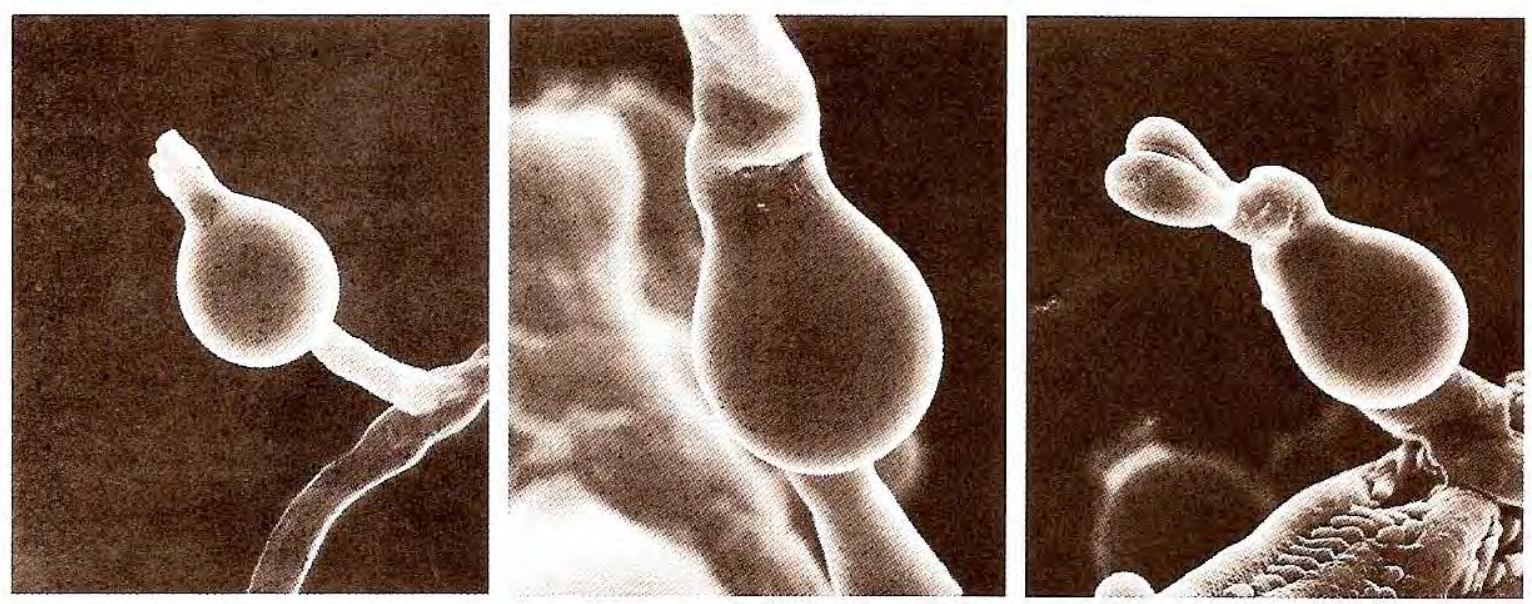

Micrografías 14-16. Cepas de Fonsecaea pedrosoi procesadas por el método 4.

\section{Discusión}

Las soluciones fijadoras convencionales utilizadas en los métodos 1,2 y 3 , además de las alteraciones presentadas, ocasionaron el fácil desprendimiento de las estructuras aéreas o conidios. La fijación con los vapores de acroleína y cristales de $\mathrm{OsO}_{4}$ (método 4) evitó la pérdida de estructuras y mantuvo los arreglos característicos intactos permitiendo obtener los detalles ultraestructurales de interés. Sumado, además, a que la acroleína reacciona y penetra más rápidamente que los otros fijadores $(10,12)$. Las alteraciones, distorsiones 0 artificios presentados en los tres primeros métodos pueden atribuirse a los efectos físicos o químicos durante una o varias de las fases del procesamiento, como ya ha sido demostrado en varios trabajos (13-16).

La esporulación tipo fiálide y Cladosporium observadas son comparables con lo descrito en trabajos al microscopio de luz (5) y al MER (7). En la conidiación tipo Cladosporium, el ápice hinchado de la célula conidiógena con dentículos se considera como una proliferación simpodial de la célula conidiógena por algunos autores (17).

La esporulación tipo Rhinocladiella ha sido motivo de controversia. Al microscopio de luz se caracteriza por tener conidióforo simpodial dematiáceo, erecto, septado, con cicatrices de donde salen los conidios (6). Los conidios nacen de una célula dematiácea, cilíndrica, que se forma a lo largo de una gran porción del raquis con un arreglo acropleurógeno sin producción de conidios secundarios (6). Publicaciones recientes presentan al género Fonsecaea con la esporulación tipo Rhinocladiella con producción de conidios secundarios (18). Este concepto amplio del género Fonsecaea se remonta a los trabajos de Schol-Schwartz y Hoog (19), donde se eliminan los géneros considerados como Rhinocladiella y se mantiene a $F$. pedrosoien el género Fonsecaea. McGinnis, Schell y Cole no describen la conidiación tipo Rhinocladiella en Fonsecaea $(6,7)$. Sin embargo, consideramos al igual que McGinnis que el género Fonsecaea debe ser definido sobre la base de su anamorfo más frecuente, que es una célula conidiógena simpodial a menudo irregular, hinchada en el ápice,de la cual se producen conidios holoblásticos primarios típicamente en dentículos, los cuales pueden convertirse en células conidiogénicas.

El arreglo de la micrografía 14 probablemente corresponde al inicio de la esporulación tipo Rhinocladiella que no ha sido documentado en observaciones al microscopio de luz o al microscopio de contraste de fase. Los arreglos de las micrografías 15 y 16 no se han informado en otros trabajos morfológicos estructurales $(6,7) \mathrm{y}$, posiblemente, corresponden a un arreglo simpodial en formación. 
En un trabajo amplio del género Fonsecaea y su relación con otros géneros (6), de 54 cepas estudiadas de $F$. pedrosoi, no se analizaron cepas colombianas. Por esta razón, sería importante analizar un mayor número de aislamientos y de diferentes regiones del país para definir si este tipo de arreglos corresponden a características aisladas propias de dos cepas (micrografías 14 a 16) o pueden encontrarse en los aislamientos colombianos. Así mismo, son necesarios más estudios al MER y al microscopio electrónico de transmisión para tratar de definir sus características funcionales y estructurales.

El análisis ultraestructural reveló detalles del proceso de conidiogénesis, cicatrices de esporulación, colarete, dentículos y conidios, mostrando arreglos característicos y sustancias que los unen que no se pueden apreciar en el microscopio de luz o en el microscopio de contraste de fase, y permite un mejor entendimiento del proceso de conidiogénesis.

La fijación con acroleína pura y cristales de $\mathrm{OsO}_{4}$ y el secado en alto vacío se pueden utilizar como una alternativa metodológica en especímenes fúngicos o no, de difícil manipulación o que presenten fácil alteración por su composición higrófila, ya que una buena interpretación de las imágenes obtenidas con el microscopio electrónico dependen en gran parte del correcto y adecuado procesamiento de la muestra a estudiar.

\section{Agradecimientos}

A la Agencia de Cooperación Internacional del Gobierno del Japón (JICA), a la Unidad de Microscopía Electrónica de la Universidad de Costa Rica y a los doctores Yutaka Futaesaku y Francisco Hernández por la información sobre los métodos empleados. A la doctora Myrtha Arango por sus valiosas sugerencias.

\section{Referencias}

1. McGinnis MR. Chromoblastomycosis and phaeohyphomycosis. New concepts, diagnosis and mycology. J Am Acad Dermatol 1983;8:1.

2. Ramírez A. Cromoblastomicosis. Revisión de 41 casos en la ciudad de Medellín, Colombia. Laboratorio \& Medicina 1994;5.

3. Padhye AA. Identification of the etiologic agents of chromoblastomycosis. In: Proceeding of the sixth International Conference on the mycoses. PAHO-WHO. Scientific Publication No. 479. Washington, D.C., 1986:87.
4. Dixon DM, Polak-Wyss A. The medically important dematiaceus fungiand their identification. Mycoses 1991;34:1.

5. Silva-Hunter M, Carrion AL. Differential characteristics of the fungal agents of chromoblastomycosis. In: Proceeding of the Third International Conference on the Mycoses. Mycoses. PAHO-WHO. Scientific Publication No. 304. Washington, D.C., 1975:118.

6. Mc Ginnis MR, Schell WA. The genus Fonsecaea and its relationship to the genera Cladosporium, Phialophora, Ranichloridium and Rhinocladiella. In: Proceeding of the Fifth International Conference on the Mycoses. Superficial, cutaneous and subcutaneous infections. PAHOWHO. Scientific Publication No. 396. Washington, D.C., 1980:215.

7. Cole GT. Conidiogenesis in the blackyeast. In:Proceeding of the Fourth International Conference on the Mycoses. The black and white teasts. PAHO-WHO. Scientific Publication No. 356. Washington, D.C., 1978:66.

8. Konemam EW, Robert G. Micología práctica de laboratorio. Tercera edición. Buenos Aires: Editorial Médica Panamericana, 1987:92.

9. Agudelo $\mathrm{CM}$, Chica J. Procesamiento de hongos para microscopía electrónica de rastreo a partir de microcultivos (tesis). Santafé de Bogotá, D.C.: Universidad de los Andes, 1992.

10. Arroyo 0. Conceptos básicos sobre el procesamiento de tejidos para microscopía electrónica. San José: Editorial de la Universidad de Costa Rica, 1991.

11. Inonue T, Osatake H. A new drying method of biological specimens for scanning electron microscopy. The t-butyl alcohol freeze-drying method. Arch Histol Cytol 1988;51:53.

12. González R. Técnicas de microscopía electrónica en biología. Barcelona: Ediciones Aguilar S.A, 1969.

13. Eskelinen S, Saukko P. Effects of glutaraldehyde and critical point drying on the shape and size of erythroccytes in isotonic and hypotonic media. J Microsc 1983;130:63.

14. Beckett A, Read ND, Porter R. Variations in fungal dimensions in relation to preparatory techniques for light microscopy and scanning electron microscopy. J Microsc 1984;136:87.

15. Boyde A, Boyde S. Further studies of specimen volume changes during processinf for SEM, including some plant tissue. Scanning Microsc 1980;II:117.

16. Nickerson A, Bulla L, Kurtzman EC. Spores. In: Hayat $M A$, eds. Principles and techniques of scanning electron microscopy. Vol I. New York: Van Nostrand Reinhold Company, 1974:159.

17. Cole GT. Conidiogenesis and conidiomatal ontogeny. In: Cole GT, Kendrick B, eds. Biology of conidial fungi. Vol 2. New York: Academic Press, 1981:288.

18. Kwon-Chung KJ, Bennett JE. Medical mycology. Philadelphia: Lea \& Febiger, 1992:347.

19. Mc Ginnis MR. Laboratory handbook of medical mycology. New York: Academic Press, 1980:261. 